\title{
SINGLETON CONDITIONS AND QUANTUM DE FINETTI'S THEOREM
}

\author{
LUIGI ACCARDI \\ CIVV Rome 2, Via Columbia, 2, 00133 Rome, Italy \\ accardi@volterra.uniroma2.it \\ ANIS BEN GHORBAL \\ Al Imam Muhammad Ibn Saud Islamic University, \\ Riyadh, Kingdom of Saudi Arabia \\ assghorbal@imamu.edu.sa \\ VITONOFRIO CRISMALE* and YUN GANG LU ${ }^{\dagger}$ \\ Dipartimento di Matematica, Università di Bari, \\ Via Orabona 4, I-70125 Bari, Italy \\ *crismalev@dm.uniba.it \\ ${ }^{\dagger} l u @ d m . u n i b a . i t$
}

Received 28 November 2007

Revised 14 October 2008

Communicated by F. Fagnola

\begin{abstract}
We investigate the validity of quantum De Finetti's Theorem for algebraic random processes satisfying the singleton condition.

We first prove a conditional version of the theorem, then we give some natural conditions which guarantee the full validity of De Finetti's Theorem.

Keywords: Algebraic stochastic process; exchangeability; singleton conditions; De Finetti's Theorem.

AMS Subject Classification: 60B99, 81S99, 46L53
\end{abstract}

\section{Introduction}

De Finetti's theorem, showing the relationship between exchangeability and stochastic independence, has inspired a large literature both in classical and quantum probability. ${ }^{5,6,9,11-14}$

The early quantum probabilistic extensions were dealing with one-dimensional index set and tensor ${ }^{27}$ or Fermi independence. ${ }^{18,19}$

Extensions to continuous index set were discussed by Freedman ${ }^{15}$ and Kallenberg $^{22}$ in the classical case and by Accardi and Lu in Ref. 4 . 
More recently a free version of De Finetti's theorem was proved by Lehner ${ }^{24}$ (see also the related paper by Köstler ${ }^{23}$ ).

Since there are many notions of stochastic independence, it would be desirable to find some general conditions, common to a multiplicity of such notions, which guarantee the validity of the thesis of De Finetti's theorem.

The notion of singleton independence is a natural candidate for this goal: even if there are notions of independence which do not satisfy this condition (see Ref. 1) for a counterexample), it is common to all the main notions of stochastic independence, including tensor, Fermi, free, monotone, boolean, ..., and it is almost sufficient to guarantee the validity of the central limit theorem.

Therefore it is natural to conjecture that it might also be almost sufficient for the validity of the thesis of De Finetti's theorem.

In this paper we give a precise statement of this conjecture and we list some natural additional conditions (which are necessary for the usual formulations of De Finetti's theorem) under which the conjecture is true.

Our main results are Theorem 3.4 and its corollaries. There are stated some conditions under which one can characterize the extremal points of the convex of exchangeable states.

An algebraic probability space is a pair $\{\mathcal{A}, \varphi\}$ where $\mathcal{A}$ is a unital *algebra and $\varphi: \mathcal{A} \rightarrow \mathbb{C}$ a state. An algebraic stochastic process is a quadruple $\left\{\{\mathcal{A}, \varphi\}, \mathcal{B},\left(j_{i}\right)_{i \in \mathcal{I}}\right\}$ where $\{\mathcal{A}, \varphi\}$ is an algebraic probability space, $\mathcal{I}$ a set, $\mathcal{B}$ a unital $*$-algebra and for any $i \in \mathcal{I}, j_{i}: \mathcal{B} \rightarrow \mathcal{A}$ a $*$-homomorphism, which will be called algebraic random variable. When for any $i \in \mathcal{I}, j_{i}\left(1_{\mathcal{B}}\right)=1_{\mathcal{A}}$ (or, equivalently, all the $*$-subalgebras $j_{i}(\mathcal{B})$ are endowed with the same unit $1_{\mathcal{A}}$ ), we speak of unital algebraic stochastic process. Throughout the paper we shall denote by $\mathcal{A}$ its restriction to the $*$-algebra generated by $\left\{j_{i}(\mathcal{B})\right\}_{i \in \mathcal{I}} \cup 1_{\mathcal{A}}$.

Recall that two algebraic stochastic processes $\left\{\left\{\mathcal{A}_{1}, \varphi_{1}\right\}, \mathcal{B},\left(j_{i}^{(1)}\right)_{i \in \mathcal{I}}\right\}$ and $\left\{\left\{\mathcal{A}_{2}, \varphi_{2}\right\}, \mathcal{B},\left(j_{i}^{(2)}\right)_{i \in \mathcal{I}}\right\}$ are said to be stochastically equivalent if their moments agree, i.e. for any $n \in \mathbb{N}, k_{1}, \ldots, k_{n} \in \mathcal{I}, b_{1}, \ldots, b_{n} \in \mathcal{B}$

$$
\varphi_{1}\left(j_{k_{n}}^{(1)}\left(b_{n}\right) \cdots j_{k_{1}}^{(1)}\left(b_{1}\right)\right)=\varphi_{2}\left(j_{k_{n}}^{(2)}\left(b_{n}\right) \cdots j_{k_{1}}^{(2)}\left(b_{1}\right)\right) .
$$

The expectation values in (1.1) are called mixed moments or correlators. Given an algebraic probability space $\{\mathcal{A}, \varphi\}$, its GNS representation is denoted by the triple $\left\{\mathcal{K}_{\varphi}, \pi_{\varphi}, \Phi\right\}$, where $\mathcal{K}_{\varphi}$ is a pre-Hilbert space, $\pi_{\varphi}$ is a *-representation of $\mathcal{A}$ in $\mathbf{H}\left(\mathcal{K}_{\varphi}\right)$, the $*$-algebra spanned by the hermitian linear operators on $\mathcal{K}_{\varphi}$, and $\Phi$ is a cyclic vector for $\left\{\pi_{\varphi}(a): a \in \mathcal{A}\right\}$. Moreover, for all $a \in$ $\mathcal{A}, \varphi(a)=\left\langle\Phi, \pi_{\varphi}(a) \Phi\right\rangle$. If $\left\{\mathcal{K}_{\varphi_{1}}, \pi_{\varphi_{1}}, \Phi_{1}\right\},\left\{\mathcal{K}_{\varphi_{2}}, \pi_{\varphi_{2}}, \Phi_{2}\right\}$ are the GNS representations corresponding respectively to two stochastically equivalent processes $\left\{\left\{\mathcal{A}_{1}, \varphi_{1}\right\}, \mathcal{B},\left(j_{i}^{(1)}\right)_{i \in \mathcal{I}}\right\},\left\{\left\{\mathcal{A}_{2}, \varphi_{2}\right\}, \mathcal{B},\left(j_{i}^{(2)}\right)_{i \in \mathcal{I}}\right\}$, there exists a unitary isomorphism (see Ref. 3 or Ref. 26 for details) $U: \mathcal{H}_{\varphi_{1}} \rightarrow \mathcal{H}_{\varphi_{2}}$ such that

$$
U \Phi_{1}=\Phi_{2}, \quad U^{*} \pi_{\varphi_{2}}\left(j_{i}^{(2)}(b)\right) U=\pi_{\varphi_{1}}\left(j_{i}^{(1)}(b)\right)
$$

for each $i \in \mathcal{I}, b \in \mathcal{B}$, where $\mathcal{H}_{\varphi_{h}}$ is the completion of $\mathcal{K}_{\varphi_{h}}, h=1,2$. 


\section{Singletons and Exchangeability}

For any arbitrary sequence of indices, the symbol " " will denote the absence of the underwritten element in the sequence. In the following, for all $n \in \mathbb{N}$ and for any choice of $i_{1}, \ldots, i_{n} \in \mathcal{I}$, if there exists $s \in\{1,2, \ldots, n\}$ such that $i_{s} \neq i_{k}$ for any $k=1, \ldots, \hat{s}, \ldots, n$, we will say $i_{s}$ is a singleton in $\left\{i_{1}, \ldots, i_{n}\right\}$ and the family $\left\{i_{1}, \ldots, i_{n}\right\}$ has a singleton.

Definition 2.1. Let be given an algebraic probability space $\{\mathcal{A}, \varphi\}$ and a family $\left\{\mathcal{A}_{i}\right\}_{i \in \mathcal{I}}$ of $*$-subalgebras in $\mathcal{A} .\left\{\mathcal{A}_{i}\right\}_{i \in \mathcal{I}}$ is said to satisfy the singleton condition (with respect to $\varphi$ ) if for any $n \geq 1$, for any choice of $i_{1}, \ldots, i_{n} \in \mathcal{I}$ such that $i_{1} \neq i_{2} \neq \cdots \neq i_{n}$

$$
\varphi\left(a_{n} \cdots a_{1}\right)=0, \quad a_{h} \in \mathcal{A}_{i_{h}}
$$

whenever $\left\{i_{1}, \ldots, i_{n}\right\}$ has a singleton $i_{s}$ and $\varphi\left(a_{s}\right)=0$. The family above is said to satisfy the strict singleton condition (with respect to $\varphi$ ) if, under the same assumptions as above

$$
\varphi\left(a_{n} \cdots a_{1}\right)=\varphi\left(a_{s}\right) \varphi\left(a_{n} \cdots \widehat{a_{s}} \cdots a_{1}\right), \quad a_{h} \in \mathcal{A}_{i_{h}} .
$$

Definition 2.2. Let an algebraic probability space $\{\mathcal{A}, \varphi\}$ and a family $\left\{\mathcal{A}_{i}\right\}_{i \in \mathcal{I}}$ of *-subalgebras in $\mathcal{A}$ be given. $\left\{\mathcal{A}_{i}\right\}_{i \in \mathcal{I}}$ is said to satisfy the block singleton condition (with respect to $\varphi$ ) if for any $n \geq 1$, for any choice of $i_{1}, \ldots, i_{n} \in \mathcal{I}$ such that $i_{1} \neq i_{2} \neq \cdots \neq i_{n}$

$$
\varphi\left(a_{n} \cdots a_{1}\right)=\varphi\left(a_{s+q} \cdots a_{s+1} a_{s}\right) \varphi\left(a_{n} \cdots \widehat{a_{s+q}} \cdots \widehat{a_{s+1}} \widehat{a_{s}} \cdots a_{1}\right), \quad a_{h} \in \mathcal{A}_{i_{h}}
$$

if, for any $j=0, \ldots, q, i_{s+j} \notin\left\{i_{1}, \ldots, \widehat{i_{s}}, \widehat{i_{s+1}}, \ldots, \widehat{i_{s+q}}, \ldots, i_{n}\right\} .\left\{i_{s}, \ldots, i_{s+q}\right\}$ is called a block singleton in $\left\{i_{1}, \ldots, i_{n}\right\}$.

Remark 2.1. In the definition above no prescriptions are given on the family $\left\{i_{s}, i_{s+1}, \ldots, i_{s+q}\right\}$, namely the indices are not assumed to be mutually different.

From now on we will call any state satisfying the singleton, strict singleton or block singleton conditions respectively a singleton, strict singleton or block singleton state.

The strict singleton condition implies the singleton condition and, if the subalgebras $\mathcal{A}_{i}$ are all endowed with the same unit of $\mathcal{A}$, say $1_{\mathcal{A}}$, is equivalent to it. To clarify the equivalence does not hold in general, we show the following example.

Example 1. Let us take

$$
\mathcal{A}:=\left\{\left(\begin{array}{ll}
x & 0 \\
0 & y
\end{array}\right): x, y \in \mathbb{C}\right\}
$$

and its $*$-subalgebras

$$
\mathcal{A}_{1}:=\left\{\left(\begin{array}{ll}
x & 0 \\
0 & 0
\end{array}\right): x \in \mathbb{C}\right\}, \mathcal{A}_{2}:=\left\{\left(\begin{array}{ll}
0 & 0 \\
0 & x
\end{array}\right): x \in \mathbb{C}\right\} .
$$


Let us define a state $\varphi$ on $\mathcal{A}$ by $\varphi(A):=\frac{1}{2} \operatorname{Tr}(A)$ for any $A \in \mathcal{A}$. For each $i=1,2$ and $A_{i} \in \mathcal{A}_{i}, \varphi\left(A_{i}\right)=0$ if and only if $A_{i}=\left(\begin{array}{ll}0 & 0 \\ 0 & 0\end{array}\right)$. Then it is easy to check that $\varphi$ is a singleton state. It is not strict singleton, in fact for any $x, y \in \mathbb{C} \backslash\{0\}$

$$
\varphi\left(\left(\begin{array}{ll}
x & 0 \\
0 & 0
\end{array}\right)\left(\begin{array}{ll}
0 & 0 \\
0 & y
\end{array}\right)\right)=0 \neq \frac{x y}{4}=\varphi\left(\left(\begin{array}{ll}
x & 0 \\
0 & 0
\end{array}\right)\right) \varphi\left(\left(\begin{array}{ll}
0 & 0 \\
0 & y
\end{array}\right)\right) .
$$

Remark 2.2. The strict singleton condition comes from the block singleton condition when $q=0$. As the definitions suggest, a strict singleton state would be not necessarily block singleton. Even though a definite answer to such a problem can not be done here, since we have not yet found a counterexample, for all the paper we will assume the two conditions are different.

The following results provide examples for singleton, strict singleton and block singleton states.

Proposition 2.1. Let $\{\mathcal{A}, \varphi\}$ and $\left\{\mathcal{A}_{i}\right\}_{i \in \mathcal{I}}$ as in Definition 2.1. Then, if $\left\{\mathcal{A}_{i}\right\}_{i \in \mathcal{I}}$ is tensor, free, boolean, monotone, symmetric projectively independent, then it satisfies the singleton condition.

Proof. The proof in the first four cases can be found in Proposition 8.14 of Ref. 17. For the last case, we recall the definition of symmetric projective independence introduced in Ref. 7. A family $\left\{\mathcal{A}_{i}\right\}_{i \in \mathcal{I}}$ of $*$-subalgebras of $\mathcal{A}$ is $\varphi$-symmetric projectively independent if for any $n \in \mathbb{N}^{*}$, any $i:\{1, \ldots, n\} \rightarrow \mathcal{I}$ with $i(h)=: i_{h}$, there exists $\omega(i) \geq 0$ such that, for any $a_{h} \in \mathcal{A}_{i_{h}}$ :

$$
\varphi\left(a_{n} \cdots a_{1}\right)=\omega(i) \prod_{j=1}^{q} \varphi\left(\prod_{k: i_{k}=\overline{i_{j}}}^{\leftarrow} a_{k}\right)
$$

where $\left\{\overline{i_{1}}, \ldots, \overline{i_{q}}\right\}$ denotes the range of $i$ and the products $\overleftarrow{\prod}$ are taken in the same order as they occur in $a_{n} \cdots a_{1}$. Then it is easy to check the singleton condition holds.

Proposition 2.2. Let $\{\mathcal{A}, \varphi\}$ and $\left\{\mathcal{A}_{i}\right\}_{i \in \mathcal{I}}$ as in Definition 2.2. Then, if $\left\{\mathcal{A}_{i}\right\}_{i \in \mathcal{I}}$ is tensor or free independent, then it satisfies the strict singleton and the block singleton conditions.

Proof. In the tensor case the thesis easily follows. For the free case the strict singleton condition can be achieved arguing as in Proposition 8.14 of Ref. 17. For the block singleton condition we have to verify that for any $n \geq 1$, for any choice of $i_{1}, \ldots, i_{n} \in \mathcal{I}, i_{1} \neq i_{2} \neq \cdots \neq i_{n}$

$$
\varphi\left(a_{n} \cdots a_{1}\right)=\varphi\left(a_{s+q} \cdots a_{s+1} a_{s}\right) \varphi\left(a_{n} \cdots \widehat{a_{s+q}} \cdots \widehat{a_{s+1}} \widehat{a_{s}} \cdots a_{1}\right), \quad a_{h} \in \mathcal{A}_{i_{h}}
$$


whenever $\left\{i_{s}, \ldots, i_{s+q}\right\}$ is a block singleton in $\left\{i_{1}, \ldots, i_{n}\right\}$. Since in the free case all the subalgebras have the same unit of $\mathcal{A}$, one can set, for any $j \neq s, s+1, \ldots, s+q$, $\overline{a_{j}}:=a_{j}-\varphi\left(a_{j}\right) 1_{\mathcal{A}}$ and $\overline{a_{s+q} \cdots a_{s+1} a_{s}}:=a_{s+q} \cdots a_{s+1} a_{s}-\varphi\left(a_{s+q} \cdots a_{s+1} a_{s}\right) 1_{\mathcal{A}}$, thus obtaining mean zero elements. By a straightforward computation one achieves

$$
\begin{aligned}
\varphi\left(a_{n} \cdots a_{1}\right)= & \varphi\left(a_{s+q} \cdots a_{s+1} a_{s}\right) \varphi\left(a_{n} \cdots \widehat{a_{s+q}} \cdots \widehat{a_{s+1}} \widehat{a_{s}} \cdots a_{1}\right) \\
& +\sum_{\substack{j=1 \\
j \notin\{s, s+1, \ldots, s+q\}}}^{n} \varphi\left(a_{j}\right) \varphi\left(\overline{a_{n}} \cdots \widehat{a_{j}} \cdots \overline{a_{1}}\right) \\
& +\sum_{\substack{j_{1}, j_{2}=1 \\
j_{i} \notin\{s, s+1, \ldots, s+q\}}}^{n} \varphi\left(a_{j_{1}}\right) \varphi\left(a_{j_{2}}\right) \varphi\left(\overline{a_{n}} \cdots \widehat{a_{j_{2}}} \cdots \widehat{a_{j_{1}}} \cdots \overline{a_{1}}\right) \\
& +\cdots+\sum_{\substack{j_{1}, j_{n-(q+1)} \\
j_{i} \notin\{s, s+1, \ldots, s+q\}}}^{n} \varphi\left(a_{j_{1}}\right) \cdots \varphi\left(a_{j_{n-(q+1)}}\right) \varphi\left(\overline{a_{s+q} \cdots a_{s+1} a_{s}}\right) \\
& +\varphi\left(\overline{a_{n}} \ldots \overline{a_{s+q} \cdots a_{s+1} a_{s}} \cdots \overline{a_{1}}\right) .
\end{aligned}
$$

Hence, for the free independence, on the right-hand side all the summands but the first vanish.

Now we can translate Definitions 2.1 and 2.2 in the context of algebraic stochastic processes. Recall the $\left\{j_{k}\right\}_{k \in \mathbb{Z}}$ are homomorphisms, then in any expectation value of the form

$$
\varphi\left(j_{k_{q}}\left(b_{q}\right) \cdots j_{k_{1}}\left(b_{1}\right)\right)
$$

we can always assume that

$$
k_{j} \neq k_{j+1}, \quad \forall j=1, \ldots, q-1 .
$$

This convention will be assumed throughout the paper and any expectation value as (2.3) will be said to be in standard form if (2.4) is satisfied.

Definition 2.3. Let $\left\{\{\mathcal{A}, \varphi\}, \mathcal{B},\left(j_{i}\right)_{i \in \mathcal{I}}\right\}$ be an algebraic stochastic process. It satisfies the singleton condition (with respect to $\varphi$ ) if for any $n \geq 1$, for any choice of $i_{1}, \ldots, i_{n} \in I$ and $b_{n}, \ldots, b_{1} \in \mathcal{B}$

$$
\varphi\left(j_{i_{n}}\left(b_{n}\right) \cdots j_{i_{1}}\left(b_{1}\right)\right)=0
$$

whenever $\left\{i_{1}, \ldots, i_{n}\right\}$ has a singleton $i_{s}$ and $\varphi\left(j_{s}\left(b_{s}\right)\right)=0$. The same process satisfies the strict singleton condition (with respect to $\varphi$ ) if

$$
\varphi\left(j_{i_{n}}\left(b_{n}\right) \cdots j_{i_{1}}\left(b_{1}\right)\right)=\varphi\left(j_{i_{s}}\left(b_{s}\right)\right) \varphi\left(j_{i_{n}}\left(b_{n}\right) \cdots \widehat{j_{i_{s}}\left(b_{s}\right)} \cdots j_{i_{1}}\left(b_{1}\right)\right)
$$

whenever $\left\{i_{1}, \ldots, i_{n}\right\}$ has a singleton $i_{s}$.

Definition 2.4. The algebraic stochastic process $\left\{\{\mathcal{A}, \varphi\}, \mathcal{B},\left(j_{i}\right)_{i \in \mathcal{I}}\right\}$ satisfies the block singleton condition if for any $n \geq 1, i_{1}, \ldots, i_{n} \in I$ and $b_{n}, \ldots, b_{1} \in \mathcal{B}$ 


$$
\begin{aligned}
\varphi\left(j_{i_{n}}\left(b_{n}\right) \cdots j_{i_{1}}\left(b_{1}\right)\right)= & \varphi\left(j_{i_{s+q}}\left(b_{s+q}\right) \cdots j_{i_{s}}\left(b_{s}\right)\right) \\
& \left.\times \varphi\left(j_{i_{n}}\left(b_{n}\right) \cdots j_{i_{s+q}} \widehat{\left(b_{s+q}\right.}\right) \cdots \widehat{j_{i_{s}}\left(b_{s}\right)} \cdots j_{i_{1}}\left(b_{1}\right)\right)
\end{aligned}
$$

if, for any $j=0, \ldots, q, i_{s+j} \notin\left\{i_{1}, \ldots, \widehat{i_{s}}, \widehat{i_{s+1}}, \ldots, \widehat{i_{s+q}}, \ldots, i_{n}\right\}$.

Remark 2.3. As already observed, the block singleton condition is stronger than the strict singleton condition and this implies the singleton condition. For unital algebraic stochastic processes the strict singleton and singleton conditions are equivalent.

Remark 2.4. In the classical case the strict singleton condition is equivalent to the stochastic independence.

Example 2. If $\left\{\{\mathcal{A}, \varphi\}, \mathcal{B},\left(j_{i}\right)_{i \in \mathcal{I}}\right\}$ is tensor or free independent, it satisfies the strict singleton condition.

The following definition introduces the fundamental concept of exchangeability for algebraic stochastic process, which plays a central role in De Finetti's theorem.

Definition 2.5. Let $\mathcal{S}_{0}=\mathcal{S}_{0}(\mathbb{Z})$ be the group of one-to-one maps $\pi: \mathbb{Z} \rightarrow \mathbb{Z}$ such that $\pi(j)=j$ for any $j \in \mathbb{Z}$ but a finite number of points. We say that an algebraic stochastic process $\left\{\{\mathcal{A}, \varphi\}, \mathcal{B},\left(j_{k}\right)_{k \in \mathbb{Z}\}}\right.$ is $\varphi$-exchangeable if it is $\mathcal{S}_{0}$-invariant, i.e. if for any $q \in \mathbb{N}, b_{q}, \ldots, b_{1} \in \mathcal{B}, k_{q}, \ldots, k_{1} \in \mathbb{Z}$ and any $\pi \in \mathcal{S}_{0}$

$$
\varphi\left(j_{k_{q}}\left(b_{q}\right) \cdots j_{k_{1}}\left(b_{1}\right)\right)=\varphi\left(j_{\pi\left(k_{q}\right)}\left(b_{q}\right) \cdots j_{\pi\left(k_{1}\right)}\left(b_{1}\right)\right) .
$$

In such a case we also say that $\varphi$ is exchangeable (with respect to the process $\left.\left\{j_{k}\right\}_{k \in \mathbb{Z}}\right)$.

Obviously exchangeability implies that all the random variables are identically distributed, i.e. for each $b \in \mathcal{B}, k, h \in \mathbb{Z}$

$$
\varphi\left(j_{k}(b)\right)=\varphi\left(j_{h}(b)\right) .
$$

Lemma 2.1. Let $\left\{\{\mathcal{A}, \varphi\}, \mathcal{B},\left(j_{k}\right)_{k \in \mathbb{Z}}\right\}$ be a $\varphi$-exchangeable algebraic stochastic process. If $\left\{k_{s_{1}}, \ldots, k_{s_{h}}\right\}$ is a block singleton in $\left\{k_{q}, \ldots, k_{1}\right\}$, then for any $l=1, \ldots, h$, any $m_{l} \in \mathbb{Z}, m_{l} \notin\left\{\left\{k_{q}, \ldots, \widehat{k_{s_{l}}}, \ldots, k_{1}\right\} \cup\left\{m_{i}: k_{s_{i}} \neq k_{s_{l}}\right\}\right\},(2.3)$ is equal to

$$
\varphi\left(j_{k_{q}}\left(b_{q}\right) \cdots j_{m_{h}}\left(b_{s_{h}}\right) \cdots j_{m_{1}}\left(b_{s_{1}}\right) \cdots j_{k_{1}}\left(b_{1}\right)\right) \text {. }
$$

Proof. For any $l=1, \ldots, h$, fix $m_{l} \in \mathbb{Z}, m_{l} \notin\left\{\left\{k_{q}, \ldots, \widehat{k_{s_{l}}}, \ldots, k_{1}\right\} \cup\left\{m_{i}: k_{s_{i}} \neq\right.\right.$ $\left.k_{s_{l}}\right\}$ \}. Let us take $\pi: \mathbb{Z} \rightarrow \mathbb{Z}$ such that

$$
\pi(r)= \begin{cases}m_{l} & \text { if } r=k_{s_{l}}, l=1, \ldots, h \\ k_{s_{l}} & \text { if } r=m_{l}, l=1, \ldots, h \\ r & \text { otherwise }\end{cases}
$$

Then $\pi \in \mathcal{S}_{0}$ and $\varphi$-exchangeability gives us the thesis. 
Corollary 2.1. Let $\left\{\{\mathcal{A}, \varphi\}, \mathcal{B},\left(j_{k}\right)_{k \in \mathbb{Z}}\right\}$ be a $\varphi$-exchangeable algebraic stochastic process. If $k_{s}$ is a singleton in $\left\{k_{q}, \ldots, k_{1}\right\}$, then for any $m \in\left\{k_{s}\right\} \cup\left(\mathbb{Z} \backslash\left\{k_{q}, \ldots, k_{1}\right\}\right)$, (2.3) is equal to

$$
\varphi\left(j_{k_{q}}\left(b_{q}\right) \cdots j_{k_{s+1}}\left(b_{s+1}\right) j_{m}\left(b_{s}\right) j_{k_{s-1}}\left(b_{s-1}\right) \cdots j_{k_{1}}\left(b_{1}\right)\right) .
$$

Proof. It is straightforward.

Proposition 2.3. Let $\left\{\{\mathcal{A}, \varphi\}, \mathcal{B},\left(j_{k}\right)_{k \in \mathbb{Z}}\right\}$ be a $\varphi$-exchangeable algebraic stochastic process. If $\left\{k_{s_{1}}, \ldots, k_{s_{h}}\right\}$ is a block singleton in $\left\{k_{q}, \ldots, k_{1}\right\}$, then (2.3) is equal to

$$
\varphi\left(j_{k_{q}}\left(b_{q}\right) \cdots\left[\frac{1}{N} \sum_{l=M}^{N+M-1}\left(j_{k_{s_{h}}+l}\left(b_{s_{h}}\right) \cdots j_{k_{s_{1}}+l}\left(b_{s_{1}}\right)\right)\right] \cdots j_{k_{1}}\left(b_{1}\right)\right),
$$

where $M:=\max \left\{\left|k_{i}-k_{j}\right|: i, j=1, \ldots, q\right\}+1$.

Proof. In fact, if $l=M$, then for each $i \in\{1, \ldots, h\}$ one has $k_{s_{i}}+M \in$ $\mathbb{Z} \backslash\left(\left\{k_{q}, \ldots, k_{1}\right\} \cup\left\{k_{s_{p}}+M: k_{s_{p}} \neq k_{s_{i}}\right\}\right)$. Hence Lemma 2.1 allows us to write $(2.3)$ as

$$
\varphi\left(j_{k_{q}}\left(b_{q}\right) \cdots j_{k_{s_{h}}+M}\left(b_{s_{h}}\right) \cdots j_{k_{s_{1}}+M}\left(b_{s_{1}}\right) \cdots j_{k_{1}}\left(b_{1}\right)\right) .
$$

Iterating the same procedure for any $l=M+1, \ldots, N+M-1$ one finds the desired equality.

Corollary 2.2. Let $\left\{\{\mathcal{A}, \varphi\}, \mathcal{B},\left(j_{k}\right)_{k \in \mathbb{Z}}\right\}$ be a $\varphi$-exchangeable algebraic stochastic process. If $k_{s}$ is a singleton in $\left\{k_{q}, \ldots, k_{1}\right\},(2.3)$ is equal to

$$
\varphi\left(j_{k_{q}}\left(b_{q}\right) \cdots j_{k_{s+1}}\left(b_{s+1}\right)\left[\frac{1}{N} \sum_{l=M}^{N+M-1} j_{k_{s}+l}\left(b_{s}\right)\right] j_{k_{s-1}}\left(b_{s-1}\right) \cdots j_{k_{1}}\left(b_{1}\right)\right)
$$

where $M:=\max \left\{\left|k_{i}-k_{j}\right|: i, j=1, \ldots, q\right\}+1$.

Proposition 2.4. Let $\left\{\{\mathcal{A}, \varphi\}, \mathcal{B},\left(j_{k}\right)_{k \in \mathbb{Z}}\right\}$ be an exchangeable algebraic stochastic process. Then $\varphi$ is translation invariant, i.e. for any $q \in \mathbb{N}$, there exists $M \in \mathbb{N}$ such that for any $r \in \mathbb{Z}$ with $|r| \geq M$, for any $k_{q}, \ldots, k_{1} \in \mathbb{Z}$ and $b_{q}, \ldots, b_{1} \in B$ one has:

$$
\varphi\left(j_{k_{q}}\left(b_{q}\right) \cdots j_{k_{1}}\left(b_{1}\right)\right)=\varphi\left(j_{k_{q}+r}\left(b_{q}\right) \cdots j_{k_{1}+r}\left(b_{1}\right)\right) .
$$

Proof. Define

$$
M:=\max \left\{\left|k_{i}-k_{l}\right|: i, l \in\{1,2, \ldots, q\}\right\}+1 .
$$

Thus, for any $r \in \mathbb{Z},|r| \geq M$ we have $k_{l}+r \notin\left\{k_{q}, \ldots, k_{1}\right\}$ for $l \in\{1,2, \ldots, q\}$. Hence the map $\pi: \mathbb{Z} \rightarrow \mathbb{Z}$ defined by

$$
\pi(m):= \begin{cases}m+r & \text { if } m \in\left\{k_{q}, \ldots, k_{1}\right\} \\ m-r & \text { if } m \in\left\{k_{q}+r, \ldots, k_{1}+r\right\} \\ m & \text { if } m \in \mathbb{Z} \backslash\left\{k_{q}+r, \ldots, k_{1}+r, k_{q}, \ldots, k_{1}\right\}\end{cases}
$$

belongs to $\mathcal{S}_{0}$ and consequently, by exchangeability, one achieves the thesis. 


\section{Ergodicity and Quantum De Finetti's Theorem}

This section is devoted to prove a quantum analogue of the De Finetti's theorem. Namely we will show that the extremal points of the convex of the exchangeable states are exactly the block singleton states. The main feature used is the ergodic decomposition theory on $C^{*}$-algebras (see Refs. 8 and 25). Therefore, from now on we need to assume that $\mathcal{A}$ is a $C^{*}$-algebra and, consequently, the GNS representation for the pair $\{\mathcal{A}, \varphi\}$ is now given by a cyclic triple $\left\{\mathcal{H}_{\varphi}, \pi_{\varphi}, \Phi\right\}$, where $\mathcal{H}_{\varphi}$ is a Hilbert space and $\pi_{\varphi}$ a $*$-representation of $\mathcal{A}$ into $\mathbf{B}\left(\mathcal{H}_{\varphi}\right)$.

Arguing as in Proposition 1.4 of Ref. 3 one achieves the following result.

Proposition 3.1. Let $\left\{\{\mathcal{A}, \varphi\}, \mathcal{B},\left(j_{k}\right)_{k \in \mathbb{Z}}\right\}$ be an exchangeable algebraic stochastic process and $\left\{\mathcal{H}_{\varphi}, \pi_{\varphi}, \Phi\right\}$ be the GNS representation of $\{\mathcal{A}, \varphi\}$. Then there exists a unitary operator $U: \mathcal{H}_{\varphi} \rightarrow \mathcal{H}_{\varphi}$ and a $*$-automorphism $u$ on $\pi_{\varphi}(\mathcal{A})$ such that

$$
\begin{aligned}
U \Phi & =\Phi, \\
u\left(\pi_{\varphi}\left(j_{k}(b)\right)\right) & :=U \pi_{\varphi}\left(j_{k}(b)\right) U^{*}=\pi_{\varphi}\left(j_{k+1}(b)\right), \quad \text { for all } b \in \mathcal{B}, k \in \mathbb{Z} .
\end{aligned}
$$

The $*$-automorphism $u$ introduced above is called the shift on the $*$-subalgebra $\pi_{\varphi}(\mathcal{A})$ of $\mathbf{B}\left(\mathcal{H}_{\varphi}\right)$.

Theorem 3.1. (Ergodic theorem) Let $\left\{\{\mathcal{A}, \varphi\}, \mathcal{B},\left(j_{k}\right)_{k \in \mathbb{Z}}\right\}$ be an exchangeable algebraic stochastic process and $\left\{\mathcal{H}_{\varphi}, \pi_{\varphi}, \Phi\right\}$ be the $G N S$ representation of $\{\mathcal{A}, \varphi\}$. The limit

$$
\lim _{N \rightarrow \infty} \frac{1}{N} \sum_{l=0}^{N-1} u^{l}=\mathbb{E}_{\infty}
$$

exists pointwise strongly on $\pi_{\varphi}(\mathcal{A})^{\prime \prime}$ and is equal to the Umegaki conditional expectation $E_{\infty}$ onto the algebra

$$
\mathcal{A}_{\infty}^{\varphi}=\mathcal{A}_{\infty}:=\left\{x \in \pi_{\varphi}(\mathcal{A})^{\prime \prime}: u(x)=x\right\} .
$$

Proof. The thesis follows from the mean ergodic theorem as stated in Theorem 2.2.1 of Refs. 20 or in Ref. 8 .

Remark 3.1. Umegaki conditional expectation was introduced in Ref. 28. A general definition in the context of $*$-algebras can be found in Ref. 2 . In the setting of $C^{*}$-algebras, a theorem due to Tamijama characterizes a Umegaki conditional expectation as a norm-one projection onto a subalgebra.

Proposition 3.2. Let $\left\{\{\mathcal{A}, \varphi\}, \mathcal{B},\left(j_{k}\right)_{k \in \mathbb{Z}}\right\}$ be an exchangeable algebraic stochastic process, $\left\{\mathcal{H}_{\varphi}, \pi_{\varphi}, \Phi\right\}$ be the $G N S$ representation of $\{\mathcal{A}, \varphi\}$ and $q \in \mathbb{N}^{*}$. If $\left\{k_{s_{1}}, \ldots, k_{s_{h}}\right\}$ is a block singleton in $\left\{k_{q}, \ldots, k_{1}\right\}$, then 


$$
\begin{aligned}
\varphi\left(j_{k_{q}}\left(b_{q}\right) \cdots j_{k_{1}}\left(b_{1}\right)\right) \\
=\left\langle\Phi, \pi_{\varphi}\left(j_{k_{q}}\left(b_{q}\right) \cdots j_{k_{s_{h}+1}}\left(b_{s_{h}+1}\right)\right) \mathbb{E}_{\infty}\left[\pi_{\varphi}\left(j_{k_{s_{h}}}\left(b_{s_{h}}\right)\right) \cdots \pi_{\varphi}\left(j_{k_{s_{1}}}\left(b_{s_{1}}\right)\right)\right]\right. \\
\left.\quad \pi_{\varphi}\left(j_{k_{s_{1}-1}}\left(b_{s_{1}-1}\right) \cdots j_{k_{1}}\left(b_{1}\right)\right) \Phi\right\rangle
\end{aligned}
$$

Proof. Notice that for any $M \geq 1$

$$
\mathbb{E}_{\infty}=\lim _{N \rightarrow \infty} \frac{1}{N} \sum_{l=M}^{N+M-1} u^{l}
$$

In fact, take $\pi_{\varphi}(a) \in \pi_{\varphi}(\mathcal{A})^{\prime \prime}$. Then

$$
\begin{aligned}
\lim _{N \rightarrow \infty} \frac{1}{N} \sum_{l=M}^{N+M-1} u^{l}\left(\pi_{\varphi}(a)\right) & =u^{M} \lim _{N \rightarrow \infty} \frac{1}{N} \sum_{l=0}^{N-1} u^{l}\left(\pi_{\varphi}(a)\right) \\
& =u^{M} \mathbb{E}_{\infty}\left(\pi_{\varphi}(a)\right)=\mathbb{E}_{\infty}\left(\pi_{\varphi}(a)\right)
\end{aligned}
$$

where the last equality is obtained since $\mathbb{E}_{\infty}\left(\pi_{\varphi}(a)\right) \in \mathcal{A}_{\infty}$. Moreover, by Proposition 2.3 ,

$$
\begin{aligned}
& \varphi\left(j_{k_{q}}\left(b_{q}\right) \cdots j_{k_{1}}\left(b_{1}\right)\right) \\
& \quad=\varphi\left(j_{k_{q}}\left(b_{q}\right) \cdots\left[\frac{1}{N} \sum_{l=M}^{N+M-1}\left(j_{k_{s_{h}}+l}\left(b_{s_{h}}\right) \cdots j_{k_{s_{1}}+l}\left(b_{s_{1}}\right)\right)\right] \cdots j_{k_{1}}\left(b_{1}\right)\right),
\end{aligned}
$$

where $M:=\max \left\{\left|k_{i}-k_{j}\right|: i, j=1, \ldots, q\right\}+1$. Using the GNS representation and exploiting the homomorphism property of $u,(3.3)$ can be written as

$$
\left\langle\Phi, \pi\left(j_{k_{q}}\left(b_{q}\right) \cdots\right)\left[\frac{1}{N} \sum_{l=M}^{N+M-1} u^{l} \pi_{\varphi}\left(j_{k_{s_{h}}}\left(b_{s_{h}}\right) \cdots j_{k_{s_{1}}}\left(b_{s_{1}}\right)\right)\right] \pi\left(\cdots j_{k_{1}}\left(b_{1}\right)\right) \Phi\right\rangle \text {. }
$$

Taking the limit for $N \rightarrow \infty$ one achieves the thesis.

Corollary 3.1. Let $\left\{\{\mathcal{A}, \varphi\}, \mathcal{B},\left(j_{k}\right)_{k \in \mathbb{Z}}\right\}$ be an exchangeable algebraic stochastic process, $\left\{\mathcal{H}_{\varphi}, \pi_{\varphi}, \Phi\right\}$ be the GNS representation of $\{\mathcal{A}, \varphi\}$ and $q \in \mathbb{N}^{*}$. If $k_{s}$ is a singleton in $\left\{k_{q}, \ldots, k_{1}\right\}$, then

$$
\begin{aligned}
& \varphi\left(j_{k_{q}}\left(b_{q}\right) \cdots j_{k_{1}}\left(b_{1}\right)\right) \\
& \quad=\left\langle\Phi, \pi_{\varphi}\left(j_{k_{q}}\left(b_{q}\right) \cdots\right) \mathbb{E}_{\infty}\left[\pi_{\varphi}\left(j_{k_{s}}\left(b_{s}\right)\right)\right] \pi_{\varphi}\left(\cdots j_{k_{1}}\left(b_{1}\right)\right) \Phi\right\rangle .
\end{aligned}
$$

Corollary 3.2. Under the same assumptions of the corollary above, if $k_{s}$ is a singleton for $\left\{k_{q}, \ldots, k_{1}\right\}$ and

$$
\mathbb{E}_{\infty}\left[\pi_{\varphi}\left(j_{k_{s}}\left(b_{s}\right)\right)\right]=0
$$

then the expectation value (3.4) is equal to 0. 
In the previous section we presented some factorization rules (i.e. singleton conditions) for joint expectations in order to achieve, in the noncommutative case, a condition replacing the stochastic independence appearing in the classical De Finetti's theorems. To gain such results we need now to get a bridge between singleton and extremal exchangeable states. The following definition should be viewed in this direction.

Definition 3.1. An exchangeable state $\varphi$ on a unital $*$-algebra $\mathcal{A}$ is called ergodic if it is extremal.

$\varphi$ is called 1-ergodic if the asymptotic algebra $\mathcal{A}_{\infty}$ is trivial, i.e.

$$
\mathcal{A}_{\infty}=\mathbb{C} \cdot 1 \text {. }
$$

The former part of definition above comes from Definition 3.1.9 of Ref. 25. The next result clarifies the emergence of the latter.

Theorem 3.2. For $\left\{\{\mathcal{A}, \varphi\}, \mathcal{B},\left(j_{k}\right)_{k \in \mathbb{Z}}\right\}$ exchangeable algebraic stochastic process, the following are equivalent

(i) $\varphi$ is 1-ergodic

(ii) $\varphi$ is a block singleton state.

Proof. (i) $\Rightarrow$ (ii). For any $n \in \mathbb{N}, b_{1}, \ldots, b_{n} \in \mathcal{B}, k_{1}, \ldots, k_{n} \in \mathbb{Z}$, the exchangeability gives

$$
\begin{aligned}
\varphi\left(j_{k_{n}}\left(b_{n}\right) \cdots j_{k_{1}}\left(b_{1}\right)\right) & =\frac{1}{N} \sum_{l=0}^{N-1} \varphi\left(j_{k_{n}+l}\left(b_{n}\right) \cdots j_{k_{1}+l}\left(b_{1}\right)\right) \\
& =\frac{1}{N} \sum_{l=0}^{N-1}\left\langle\Phi, u^{l} \pi_{\varphi}\left(j_{k_{n}}\left(b_{n}\right) \cdots j_{k_{1}}\left(b_{1}\right)\right) \Phi\right\rangle .
\end{aligned}
$$

Taking the limit for $N \rightarrow \infty$ in (3.5) and using Theorem 3.1 one has

$$
\varphi\left(j_{k_{n}}\left(b_{n}\right) \cdots j_{k_{1}}\left(b_{1}\right)\right)=\left\langle\Phi, \mathbb{E}_{\infty}\left(\pi_{\varphi}\left(j_{k_{n}}\left(b_{n}\right) \cdots j_{k_{1}}\left(b_{1}\right)\right)\right) \Phi\right\rangle .
$$

Hence, the state being 1-ergodic,

$$
\mathbb{E}_{\infty}\left(\pi_{\varphi}\left(j_{k_{n}}\left(b_{n}\right) \cdots j_{k_{1}}\left(b_{1}\right)\right)\right)=\varphi\left(j_{k_{n}}\left(b_{n}\right) \cdots j_{k_{1}}\left(b_{1}\right)\right) \cdot 1
$$

Let $q \in \mathbb{N}^{*}$ and take $\left\{k_{s_{1}}, \ldots, k_{s_{h}}\right\}$ a block singleton in $\left\{k_{1}, \ldots, k_{q}\right\}$. For any $b_{1}, \ldots, b_{q} \in \mathcal{B},(3.2)$ gives

$$
\begin{aligned}
\varphi\left(j_{k_{q}}\left(b_{q}\right) \cdots j_{k_{s_{h}}}\left(b_{s_{h}}\right) \cdots j_{k_{s_{1}}}\left(b_{s_{1}}\right) \cdots j_{k_{1}}\left(b_{1}\right)\right) \\
=\left\langle\Phi, \pi_{\varphi}\left(j_{k_{q}}\left(b_{q}\right) \cdots j_{k_{s_{h}+1}}\left(b_{s_{h}+1}\right)\right) \mathbb{E}_{\infty}\left[\pi_{\varphi}\left(j_{k_{s_{h}}}\left(b_{s_{h}}\right)\right) \cdots \pi_{\varphi}\left(j_{k_{s_{1}}}\left(b_{s_{1}}\right)\right)\right]\right. \\
\left.\quad \pi_{\varphi}\left(j_{k_{s_{1}-1}}\left(b_{s_{1}-1}\right) \cdots j_{k_{1}}\left(b_{1}\right)\right) \Phi\right\rangle .
\end{aligned}
$$

From (3.6), we can write the quantity above as

$$
\left.\left.\varphi\left(j_{k_{s_{h}}}\left(b_{s_{h}}\right) \cdots j_{k_{s_{1}}}\left(b_{s_{1}}\right)\right) \varphi\left(j_{k_{q}}\left(b_{q}\right) \cdots j_{k_{s_{h}}\left(b_{s_{h}}\right.}\right) \cdots \widehat{j_{k_{s_{1}}}\left(b_{s_{1}}\right.}\right) \cdots j_{k_{1}}\left(b_{1}\right)\right) .
$$


(ii) $\Rightarrow$ (i). Let us take $a \in \mathcal{A}$. Since $\mathcal{A}$ is generated by $\left\{j_{k}(\mathcal{B})\right\}_{k \in \mathbb{Z}} \cup 1_{\mathcal{A}}$, then $a=j_{k_{h}}\left(b_{h}\right) \cdots j_{k_{1}}\left(b_{1}\right)$ for some $h \geq 1, b_{1}, \ldots, b_{h} \in \mathcal{B}, k_{1}, \ldots, k_{h} \in \mathbb{Z}$.

For $\xi:=\pi_{\varphi}\left(j_{\widetilde{k_{q}}}\left(\widetilde{b}_{q}\right) \cdots j_{\widetilde{k_{1}}}\left(\tilde{b}_{1}\right)\right) \Phi, \eta:=\pi_{\varphi}\left(j_{\overline{k_{r}}}\left(\overline{b_{r}}\right) \cdots j_{\overline{k_{1}}}\left(\overline{b_{1}}\right)\right) \Phi$ arbitrary vectors in $\pi_{\varphi}(\mathcal{A}) \Phi$

$$
\begin{aligned}
\left\langle\eta, \mathbb{E}_{\infty}\right. & \left.\left(\pi_{\varphi}(a)\right) \xi\right\rangle \\
= & \left\langle\Phi, \pi_{\varphi}\left(j_{\overline{k_{1}}}\left({\overline{b_{1}}}^{*}\right) \cdots j_{\bar{k}_{r}}\left({\overline{b_{r}}}^{*}\right)\right) \mathbb{E}_{\infty}\left(\pi_{\varphi}\left(j_{k_{h}}\left(b_{h}\right) \cdots j_{k_{1}}\left(b_{1}\right)\right)\right)\right. \\
& \left.\pi_{\varphi}\left(j_{\widetilde{k_{q}}}\left(\widetilde{b_{q}}\right) \cdots j_{\widetilde{k_{1}}}\left(\tilde{b}_{1}\right)\right) \Phi\right\rangle .
\end{aligned}
$$

Let us firstly assume that $\left\{k_{1}, \ldots, k_{h}\right\}$ is a block singleton in

$$
K:=\left\{\overline{k_{1}}, \ldots, \overline{k_{r}}, k_{1}, \ldots, k_{h}, \widetilde{k_{1}}, \ldots, \widetilde{k_{q}}\right\},
$$

then, by Proposition 3.2 and the block singleton condition, (3.7) is equal to

$$
\begin{aligned}
& \varphi\left(j_{\bar{k}_{1}}\left({\overline{b_{1}}}^{*}\right) \cdots j_{{\overline{k_{r}}}}\left({\overline{b_{r}}}^{*}\right) j_{k_{h}}\left(b_{h}\right) \cdots j_{k_{1}}\left(b_{1}\right) j_{\widetilde{k_{q}}}\left(\widetilde{b_{q}}\right) \cdots j_{\widetilde{k_{1}}}\left(\widetilde{b_{1}}\right)\right) \\
&= \varphi\left(j_{k_{h}}\left(b_{h}\right) \cdots j_{k_{1}}\left(b_{1}\right)\right) \\
&\left.\times \varphi\left(j_{\overline{k_{1}}}\left({\overline{b_{1}}}^{*}\right) \cdots j_{\overline{k_{r}}}\left({\overline{b_{r}}}^{*}\right) \widehat{j_{k_{h}}\left(b_{h}\right.}\right) \cdots \widehat{j_{k_{1}}\left(b_{1}\right)} j_{\widetilde{k_{q}}}\left(\widetilde{b_{q}}\right) \cdots j_{\widetilde{k_{1}}}\left(\widetilde{b_{1}}\right)\right) .
\end{aligned}
$$

Notice that (3.8) is nothing but

$$
\varphi(a)\langle\eta, \xi\rangle
$$

From the density of $\pi_{\varphi}(\mathcal{A}) \Phi$ into $\mathcal{H}_{\varphi}$, one achieves $\mathbb{E}_{\infty}\left(\pi_{\varphi}(a)\right)=\varphi(a) \cdot 1$.

If $\left\{k_{1}, \ldots, k_{h}\right\}$ is not a block singleton in $K$, then for $M:=\max _{k_{i}, k_{j} \in K}\left\{\mid k_{i}-\right.$ $\left.k_{j} \mid\right\}+1$, exchangeability ensures us that

$$
\varphi(a) \cdot 1=\varphi\left(j_{k_{h}+M}\left(b_{h}\right) \cdots j_{k_{1}+M}\left(b_{1}\right)\right) \cdot 1
$$

and $\left\{k_{1}+M, \ldots, k_{h}+M\right\}$ becomes a block singleton in $\left\{\overline{k_{1}}, \ldots, \overline{k_{r}}, k_{1}+M, \ldots, k_{h}+\right.$ $\left.M, \widetilde{k_{1}}, \ldots, \widetilde{k_{q}}\right\}$. Therefore, arguing as above, one finds

$$
\varphi\left(j_{k_{h}+M}\left(b_{h}\right) \cdots j_{k_{1}+M}\left(b_{1}\right)\right) \cdot 1=\mathbb{E}_{\infty}\left(\pi_{\varphi}\left(j_{k_{h}+M}\left(b_{h}\right) \cdots j_{k_{1}+M}\left(b_{1}\right)\right)\right)
$$

on the whole $\mathcal{H}_{\varphi}$. The right-hand side above can be written as

$$
\mathbb{E}_{\infty} u^{M}\left(\pi_{\varphi}\left(j_{k_{h}}\left(b_{h}\right) \cdots j_{k_{1}}\left(b_{1}\right)\right)\right)
$$

since, $u$ and $\mathbb{E}_{\infty}$ are mutually commutative, one obtains

$$
\varphi\left(j_{k_{h}+M}\left(b_{h}\right) \cdots j_{k_{1}+M}\left(b_{1}\right)\right) \cdot 1=\mathbb{E}_{\infty}\left(\pi_{\varphi}\left(j_{k_{h}}\left(b_{h}\right) \cdots j_{k_{1}}\left(b_{1}\right)\right)\right) .
$$

Then, by (3.9), one ends the proof.

Corollary 3.3. Let $\left\{\{\mathcal{A}, \varphi\}, \mathcal{B},\left(j_{k}\right)_{k \in \mathbb{Z}}\right\}$ be an exchangeable algebraic stochastic process. If $\varphi$ is 1-ergodic, then it is a strict singleton state.

Proof. Clear. 
The 1-ergodicity is not a consequence of the strict singleton condition. In fact, using the notations introduced above, if $a=j_{k_{h}}\left(b_{h}\right) \cdots j_{k_{1}}\left(b_{1}\right)$ and $\left\{k_{1}, \ldots, k_{h}\right\}$ has not singletons but is a block singleton in $K$, one cannot pull out the "block singleton" part from the following mixed moment

$$
\varphi\left(j_{\overline{k_{1}}}\left({\overline{b_{1}}}^{*}\right) \cdots j_{\overline{k_{r}}}\left({\overline{b_{r}}}^{*}\right) j_{k_{h}}\left(b_{h}\right) \cdots j_{k_{1}}\left(b_{1}\right) j_{{\widetilde{k_{q}}}_{q}}\left(\widetilde{b_{q}}\right) \cdots j_{\widetilde{k_{1}}}\left(\widetilde{b_{1}}\right)\right) .
$$

Hence there is no hope to understand if $\mathbb{E}_{\infty}\left(\pi_{\varphi}(a)\right)$ is, up to a multiplicative constant, the identity operator on $\mathcal{H}_{\varphi}$. If one aims to use only the strict singleton condition as a factorization rule for the mixed moments, and contemporarily obtain information about the structure of the asymptotic algebra, some additional conditions should be done on the algebra $\mathcal{A}$. The following lemma suggests a possible way to get such conditions.

Lemma 3.1. Let $\left\{\{\mathcal{A}, \varphi\}, \mathcal{B},\left(j_{k}\right)_{k \in \mathbb{Z}}\right\}$ be an exchangeable algebraic stochastic process satisfying the strict singleton condition and $\left\{\mathcal{H}_{\varphi}, \pi_{\varphi}, \Phi\right\}$ be the GNS representation of $\{\mathcal{A}, \varphi\}$. For any $j_{k_{n}}\left(b_{n}\right) \cdots j_{k_{1}}\left(b_{1}\right), n \in \mathbb{N}, k_{n}>\cdots>k_{1} \in \mathbb{N}, b_{1}, \ldots, b_{n} \in \mathcal{B}$, for any $\xi, \eta \in \pi_{\varphi}(\mathcal{A}) \Phi$, one has

$$
\begin{gathered}
\left\langle\eta, \mathbb{E}_{\infty}\left(\pi_{\varphi}\left(j_{k_{n}}\left(b_{n}\right)\right)\right) \cdots \mathbb{E}_{\infty}\left(\pi_{\varphi}\left(j_{k_{1}}\left(b_{1}\right)\right)\right) \xi\right\rangle \\
=\left\langle\eta, \mathbb{E}_{\infty}\left(\pi_{\varphi}\left(j_{k_{n}}\left(b_{n}\right) \cdots j_{k_{1}}\left(b_{1}\right)\right)\right) \xi\right\rangle
\end{gathered}
$$

Proof. Let us first compute, for any $N \in \mathbb{N}$,

$$
\left\langle\eta, \frac{1}{N^{n}} \sum_{l_{n}=0}^{N-1} u^{l_{n}}\left(\pi_{\varphi}\left(j_{k_{n}}\left(b_{n}\right)\right)\right) \cdots \sum_{l_{1}=0}^{N-1} u^{l_{1}}\left(\pi_{\varphi}\left(j_{k_{1}}\left(b_{1}\right)\right)\right) \xi\right\rangle .
$$

It can be written as

$$
\begin{aligned}
\langle\eta, & \left.\frac{1}{N^{n}} \sum_{l_{n}=0}^{N-1} \pi_{\varphi}\left(j_{k_{n}+l_{n}}\left(b_{n}\right)\right) \cdots \sum_{l_{1}=0}^{N-1} \pi_{\varphi}\left(j_{k_{1}+l_{1}}\left(b_{1}\right)\right) \xi\right\rangle \\
= & \left\langle\eta, \frac{1}{N^{n}} \sum_{l_{n}=0}^{N-1} \pi_{\varphi}\left(j_{k_{n}+l_{n}}\left(b_{n}\right)\right)\right. \\
& \times\left[\sum_{\substack{l_{n-1}=0 \\
k_{n-1}+l_{n-1} \notin\left\{k_{n}+l_{n}\right\}}}^{N-1} \pi_{\varphi}\left(j_{k_{n-1}+l_{n-1}}\left(b_{n-1}\right)\right)+\left(\pi_{\varphi}\left(j_{k_{n}+l_{n}}\left(b_{n-1}\right)\right)\right]\right. \\
& \left.\ldots \sum_{l_{1}=0}^{N-1} \pi_{\varphi}\left(j_{k_{1}+l_{1}}\left(b_{1}\right)\right) \xi\right\rangle
\end{aligned}
$$




$$
\begin{aligned}
& =\cdots=\left\langle\eta, \frac{1}{N^{n}} \sum_{l_{n}=0}^{N-1} \pi_{\varphi}\left(j_{k_{n}+l_{n}}\left(b_{n}\right)\right)\right. \\
& \times\left[\sum_{\substack{l_{n-1}=0 \\
k_{n-1}+l_{n-1} \notin\left\{k_{n}+l_{n}\right\}}}^{N-1} \pi_{\varphi}\left(j_{k_{n-1}+l_{n-1}}\left(b_{n-1}\right)\right)+\pi_{\varphi}\left(j_{k_{n}+l_{n}}\left(b_{n-1}\right)\right)\right] \\
& {\left[\sum_{\substack{l_{1}=0 \\
k_{1}+l_{1} \notin\left\{k_{n}+l_{n}, \ldots, k_{2}+l_{2}\right\}}}^{N-1} \pi_{\varphi}\left(j_{k_{1}+l_{1}}\left(b_{1}\right)\right)+\pi_{\varphi}\left(j_{k_{n}+l_{n}}\left(b_{n-1}\right)\right)\right.} \\
& \left.\left.+\cdots+\pi_{\varphi}\left(j_{k_{2}+l_{2}}\left(b_{1}\right)\right)\right] \xi\right\rangle \text {. }
\end{aligned}
$$

After computing the products appearing in the last equality of (3.11), one recognizes only one term has $n$ summations, namely

$$
\begin{gathered}
\left\langle\eta, \frac{1}{N^{n}} \sum_{l_{n}=0}^{N-1} \pi_{\varphi}\left(j_{k_{n}+l_{n}}\left(b_{n}\right)\right) \sum_{\substack{l_{n-1}=0 \\
k_{n-1}+l_{n-1} \notin\left\{k_{n}+l_{n}\right\}}}^{N-1} \pi_{\varphi}\left(j_{k_{n-1}+l_{n-1}}\left(b_{n-1}\right)\right)\right. \\
\left.\ldots \quad \sum_{\substack{l_{1}=0 \\
k_{1}+l_{1} \notin\left\{k_{n}+l_{n}, \ldots, k_{2}+l_{2}\right\}}}^{N-1} \pi_{\varphi}\left(j_{k_{1}+l_{1}}\left(b_{1}\right)\right) \xi\right\rangle
\end{gathered}
$$

whereas the remaining $n !-1$ terms have at most $n-1$ summations. Taking the limit for $N \rightarrow \infty$, all such terms tends to 0 . Hence (3.10) becomes equal to

$$
\begin{aligned}
& \left\langle\eta, \frac{1}{N^{n}} \sum_{l_{n}=0}^{N-1} \pi_{\varphi}\left(j_{k_{n}+l_{n}}\left(b_{n}\right)\right) \sum_{\substack{l_{n-1}=0 \\
k_{n-1}+l_{n-1} \notin\left\{k_{n}+l_{n}\right\}}}^{N-1} \pi_{\varphi}\left(j_{k_{n-1}+l_{n-1}}\left(b_{n-1}\right)\right)\right. \\
& \left.\ldots \quad \sum_{k_{1}+l_{1} \notin\left\{k_{n}+l_{n}, \ldots, k_{2}+l_{2}\right\}}^{N-1} \pi_{\varphi}\left(j_{k_{1}+l_{1}}\left(b_{1}\right)\right) \xi\right\rangle+O\left(\frac{1}{N}\right) .
\end{aligned}
$$

Denote $\xi:=\pi_{\varphi}\left(j_{\widetilde{k_{q}}}\left(\widetilde{b_{q}}\right) \cdots j_{\widetilde{k_{1}}}\left(\tilde{b}_{1}\right)\right) \Phi, \eta:=\pi_{\varphi}\left(j_{\overline{k_{r}}}\left(\overline{b_{r}}\right) \cdots j_{\overline{k_{1}}}\left(\overline{b_{1}}\right)\right) \Phi$. We can even suppose $\left\{k_{i}+l_{i}: l_{i}=0, \ldots, N-1, k_{i}+l_{i} \notin\left\{k_{n}+l_{n}, \ldots, k_{i+1}+l_{i+1}\right\}\right\}_{i=1}^{n}$ is a set of singletons in $\left\{\overline{k_{1}}, \ldots, \overline{k_{r}}, k_{1}, k_{1}+1, \ldots, k_{n}+N-1, \widetilde{k_{1}}, \ldots, \widetilde{k_{q}}\right\}$. In fact, in the contrary case, we properly shift them and, using the same arguments developed in the proof of Theorem 3.2, reduce to this case. By the strict singleton condition, one 
finds (3.12) is nothing else than

$$
\begin{aligned}
& \frac{1}{N^{n}} \sum_{l_{n}=0}^{N-1} \sum_{\substack{l_{n-1}=0 \\
k_{n-1}+l_{n-1} \notin\left\{k_{n}+l_{n}\right\}}}^{N-1} \cdots \sum_{\substack{l_{1}=0 \\
k_{1}+l_{1} \notin\left\{k_{n}+l_{n}, \ldots, k_{2}+l_{2}\right\}}}^{N-1} \varphi\left(j_{k_{n}+l_{n}}\left(b_{n}\right)\right) \cdots \varphi\left(j_{k_{1}+l_{1}}\left(b_{1}\right)\right) \\
& \left.\left.\quad \times \varphi\left(j_{\overline{k_{1}}}\left({\overline{b_{1}}}^{*}\right) \cdots j_{\overline{k_{r}}}\left({\overline{b_{r}}}^{*}\right) \widehat{j_{k_{n}+l_{n}}\left(b_{n}\right.}\right) \cdots \widehat{j_{k_{1}+l_{1}}\left(b_{1}\right.}\right) j_{\widetilde{k}_{q}}\left(\widetilde{b}_{q}\right) \cdots j_{\widetilde{k_{1}}}\left(\widetilde{b_{1}}\right)\right)+O\left(\frac{1}{N}\right) .
\end{aligned}
$$

For each $i=1, \ldots, n-1$, by exchangeability follows

$$
\begin{gathered}
\sum_{\substack{l_{i}=0 \\
k_{i}+l_{i} \notin\left\{k_{n}+l_{n}, \ldots, k_{i+1}+l_{i+1}\right\}}}^{N-1} \varphi\left(j_{k_{i}+l_{i}}\left(b_{i}\right)\right) \\
=\left(N-\left|\left\{k_{i}+l_{i}=k_{n}+l_{n}, \ldots, k_{i+1}+l_{i+1}\right\}\right|\right) \varphi\left(j_{m_{i}+l_{n}}\left(b_{i}\right)\right),
\end{gathered}
$$

where $|\cdot|$ denotes the cardinality. For a suitable $m_{i} \in \mathbb{Z}$, namely $m_{i} \geq$ $\max _{h, j \in\{1, \ldots, n\}}\left\{\left|k_{h}-k_{j}\right|\right\}+i$, denoting $K_{i}:=\left|\left\{k_{i}+l_{i}=k_{n}+l_{n}, \ldots, k_{i+1}+l_{i+1}\right\}\right|$, (3.13) is equal to

$$
\begin{aligned}
& \frac{\left(N-K_{n-1}\right)\left(N-K_{n-2}\right) \cdots\left(N-K_{1}\right)}{N^{n}} \sum_{l_{n}=0}^{N-1} \varphi\left(j_{k_{n}+l_{n}}\left(b_{n}\right)\right) \varphi\left(j_{m_{n-1}+l_{n}}\left(b_{n-1}\right)\right) \\
& \cdots \varphi\left(j_{m_{1}+l_{n}}\left(b_{1}\right)\right) \varphi\left(j_{\bar{k}_{1}}\left({\overline{b_{1}}}^{*}\right) \cdots j_{\overline{k_{r}}}\left({\overline{b_{r}}}^{*}\right) j_{k_{n}+l_{n}\left(b_{n}\right.}\right) \\
& \left.\cdots j_{k_{1}+l_{1}}\left(b_{1}\right) j_{\widetilde{k_{q}}}\left(\widetilde{b_{q}}\right) \cdots j_{\widetilde{k_{1}}}\left(\widetilde{b_{1}}\right)\right)+O\left(\frac{1}{N}\right) \text {. }
\end{aligned}
$$

Since exchangeability implies all the random variables are identically distributed, the quantity above is equal to

$$
\begin{aligned}
& \frac{\left(N-K_{n-1}\right)\left(N-K_{n-2}\right) \cdots\left(N-K_{1}\right)}{N^{n}} \sum_{l_{n}=0}^{N-1} \varphi\left(j_{k_{n}+l_{n}}\left(b_{n}\right)\right) \varphi\left(j_{k_{n-1}+l_{n}}\left(b_{n-1}\right)\right) \\
& \cdots \varphi\left(j_{k_{1}+l_{n}}\left(b_{1}\right)\right) \varphi\left(j_{\overline{k_{1}}}\left({\overline{b_{1}}}^{*}\right) \cdots j_{\overline{k_{r}}}\left({\overline{b_{r}}}^{*}\right) \bar{j}_{k_{n}+l_{n}}\left(b_{n}\right)\right. \\
& \left.\cdots j_{k_{1}+l_{1}}\left(b_{1}\right) j_{\widetilde{k_{q}}}\left({\widetilde{b_{q}}}\right) \cdots j_{\widetilde{k_{1}}}\left(\widetilde{b_{1}}\right)\right)+O\left(\frac{1}{N}\right) \\
& =\frac{\left(N-K_{n-1}\right)\left(N-K_{n-2}\right) \cdots\left(N-K_{1}\right)}{N^{n}} \sum_{l_{n}=0}^{N-1}\left\langle\eta, \pi_{\varphi}\left(j_{k_{n}+l_{n}}\left(b_{n}\right)\right.\right. \\
& \left.\left.\quad \cdots j_{k_{1}+l_{n}}\left(b_{1}\right)\right) \xi\right\rangle+O\left(\frac{1}{N}\right)
\end{aligned}
$$


where in the last equality we used the strict singleton condition. The right-hand side in (3.14) is equal to (3.10), namely

$$
\begin{aligned}
\langle\eta, & \left.\frac{1}{N^{n}} \sum_{l_{n}=0}^{N-1} u^{l_{n}}\left(\pi_{\varphi}\left(j_{k_{n}}\left(b_{n}\right)\right)\right) \cdots \sum_{l_{1}=0}^{N-1} u^{l_{1}}\left(\pi_{\varphi}\left(j_{k_{1}}\left(b_{1}\right)\right)\right) \xi\right\rangle \\
= & \frac{\left(N-K_{n-1}\right)\left(N-K_{n-2}\right) \cdots\left(N-K_{1}\right)}{N^{n}} \sum_{l_{n}=0}^{N-1}\left\langle\eta, \pi_{\varphi}\left(j_{k_{n}+l_{n}}\left(b_{n}\right)\right.\right. \\
& \left.\left.\cdots j_{k_{1}+l_{n}}\left(b_{1}\right)\right) \xi\right\rangle+O\left(\frac{1}{N}\right)
\end{aligned}
$$

the thesis follows after taking the limit for $N \rightarrow \infty$.

If $\mathcal{A}$ is such that the products $j_{k_{n}}\left(b_{n}\right) \cdots j_{k_{1}}\left(b_{1}\right), n \in \mathbb{N}, k_{n}>\cdots>k_{1} \in \mathbb{N}$, $b_{1}, \ldots, b_{n} \in \mathcal{B}$ are total, the strict singleton condition implies the 1-ergodicity for exchangeable states, as shown in the following result. If the condition above holds, we say $\mathcal{A}$ satisfies condition TOD (the totally ordered products are dense).

Proposition 3.3. Let $\left\{\{\mathcal{A}, \varphi\}, \mathcal{B},\left(j_{k}\right)_{k \in \mathbb{Z}}\right\}$ be an exchangeable algebraic stochastic process and suppose $\mathcal{A}$ satisfies condition TOD. Then, if $\varphi$ is a strict singleton state, it is 1-ergodic.

Proof. Let us fix $a \in \mathcal{A}$. We need to prove that there exists $\alpha \in \mathbb{C}$ such that $\mathbb{E}_{\infty}\left(\pi_{\varphi}(a)\right)=\alpha \cdot 1$. Because of the condition TOD, it is sufficient to prove it for $a=j_{k_{n}}\left(b_{n}\right) \cdots j_{k_{1}}\left(b_{1}\right), n \in \mathbb{N}, k_{n}>\cdots>k_{1} \in \mathbb{N}, b_{1}, \ldots, b_{n} \in \mathcal{B}$. As usual, denote $\xi:=\pi_{\varphi}\left(j_{\widetilde{k_{q}}}\left(\widetilde{b_{q}}\right) \cdots j_{\widetilde{k_{1}}}\left(\tilde{b}_{1}\right)\right) \Phi, \eta:=\pi_{\varphi}\left(j_{\overline{k_{r}}}\left(\overline{b_{r}}\right) \cdots j_{\overline{k_{1}}}\left(\overline{b_{1}}\right)\right) \Phi$, arbitrary vectors in $\pi_{\varphi}(\mathcal{A}) \Phi$. Then

$$
\begin{aligned}
\left\langle\eta, \mathbb{E}_{\infty}\left(\pi_{\varphi}(a)\right) \xi\right\rangle & =\left\langle\eta, \mathbb{E}_{\infty}\left(\pi_{\varphi}\left(j_{k_{n}}\left(b_{n}\right) \cdots j_{k_{1}}\left(b_{1}\right)\right)\right) \xi\right\rangle \\
& =\left\langle\eta, \mathbb{E}_{\infty}\left(\pi_{\varphi}\left(j_{k_{n}}\left(b_{n}\right)\right)\right) \cdots \mathbb{E}_{\infty}\left(\pi_{\varphi}\left(j_{k_{1}}\left(b_{1}\right)\right)\right) \xi\right\rangle
\end{aligned}
$$

where the last equality comes from Lemma 3.1.

As already observed, without loss of generality, we take $\left\{k_{1}, \ldots, k_{n}\right\}$ all singletons in $\left\{\overline{k_{1}}, \ldots, \overline{k_{r}}, k_{1}, \ldots, k_{n}, \widetilde{k_{1}}, \ldots, \widetilde{k_{q}}\right\}$. Applying Corollary $3.1,(3.15)$ is equal to

$$
\begin{aligned}
& \varphi\left(j_{\overline{k_{1}}}\left({\overline{b_{1}}}^{*}\right) \cdots j_{\overline{k_{r}}}\left({\overline{b_{r}}}^{*}\right) j_{k_{n}}\left(b_{h}\right) \cdots j_{k_{1}}\left(b_{1}\right) j_{\widetilde{k_{q}}}\left(\widetilde{b_{q}}\right) \cdots j_{\widetilde{k_{1}}}\left(\widetilde{b_{1}}\right)\right) \\
&= \varphi\left(j_{k_{n}}\left(b_{n}\right)\right) \cdots \varphi\left(j_{k_{1}}\left(b_{1}\right)\right) \\
&\left.\times \varphi\left(j_{\overline{k_{1}}}\left({\overline{b_{1}}}^{*}\right) \cdots j_{\overline{k_{r}}}\left({\overline{b_{r}}}^{*}\right) \widehat{j_{k_{n}}\left(b_{n}\right.}\right) \cdots \widehat{j_{k_{1}}\left(b_{1}\right)} j_{\widetilde{k_{q}}}\left(\widetilde{b_{q}}\right) \cdots j_{\widetilde{k_{1}}}\left(\widetilde{b_{1}}\right)\right)
\end{aligned}
$$

by the strict singleton condition. The right-hand side above is

$$
\varphi(a)\langle\eta, \xi\rangle \text {. }
$$


Since it is equal to

$$
\left\langle\eta, \mathbb{E}_{\infty}\left(\pi_{\varphi}(a)\right) \xi\right\rangle
$$

one finds $\alpha=\varphi\left(j_{k_{n}}\left(b_{n}\right)\right) \cdots \varphi\left(j_{k_{1}}\left(b_{1}\right)\right)$ from the density of $\pi_{\varphi}(\mathcal{A}) \Phi$ into $\mathcal{H}_{\varphi}$.

As a consequence of the above result, under exchangeability and the TOD conditions, strict singleton states are equivalent to 1-ergodic.

Hence a link between singleton conditions and asymptotic algebra has been established. Now we investigate their possible links with ergodic (i.e. extremal) states. A possible way is to obtain a quantum version of the celebrated lemma of Hewitt and Savage. To this aim, let us consider $\left\{\{\mathcal{A}, \varphi\}, \mathcal{B},\left(j_{k}\right)_{k \in \mathbb{Z}}\right\}$ an exchangeable algebraic stochastic process and $\left\{\mathcal{H}_{\varphi}, \pi_{\varphi}, \Phi\right\}$ the GNS representation of $\{\mathcal{A}, \varphi\}$. The exchangeability condition implies that, given an arbitrary $\sigma \in \mathcal{S}_{0}$, the algebraic stochastic process $\left\{\{\mathcal{A}, \varphi\}, \mathcal{B},\left(j_{k}^{(1)}\right)_{k \in \mathbb{Z}\}}\right.$, where $j_{k}^{(1)}:=j_{\sigma(k)}$ for all $k \in \mathbb{Z}$ is stochastically equivalent to the previous one. Hence we know there exists a unitary isomorphism $U_{\sigma}: \mathcal{H}_{\varphi} \rightarrow \mathcal{H}_{\varphi}$ such that $U_{\sigma} \Phi=\Phi$ and for any $b \in \mathcal{B}, k \in \mathbb{Z}$

$$
U_{\sigma} \pi_{\varphi}\left(j_{k}(b)\right)=\pi_{\varphi}\left(j_{\sigma(k)}(b)\right) U_{\sigma} .
$$

Moreover, the map $u_{\sigma}$ on $\pi_{\varphi}(\mathcal{A})$ such that for any $b \in \mathcal{B}, k \in \mathbb{Z}$

$$
u_{\sigma} \pi_{\varphi}\left(j_{k}(b)\right):=U_{\sigma} \pi_{\varphi}\left(j_{k}(b) U_{\sigma}^{*}=\pi_{\varphi}\left(j_{\sigma(k)}(b)\right)\right.
$$

is a $*$-automorphism. Define the symmetric algebra

$$
\begin{gathered}
\mathcal{A}_{\mathcal{S}_{0}}=\mathcal{A}_{\mathcal{S}_{0}}^{\varphi}:=\left\{x \in \pi_{\varphi}(\mathcal{A})^{\prime \prime}: u_{\sigma}(x)=x \text { for all } \sigma \in \mathcal{S}_{0}\right\}, \\
E_{\mathcal{S}_{0}}:=\left\{\xi \in \mathcal{H}_{\varphi}: U_{\sigma} \xi=\xi \text { for all } \sigma \in \mathcal{S}_{0}\right\} .
\end{gathered}
$$

Then $E_{\mathcal{S}_{0}}$ is a closed subspace of $\mathcal{H}_{\varphi}$.

Given a family of classical exchangeable random variables on a probability space, the symmetric $\sigma$-algebra coincide with the asymptotic one. This result, known as Lemma of Hewitt-Savage (see Refs. 10 and 16), has a quantum analogue in the following result.

Proposition 3.4. (Quantum Hewitt-Savage Lemma) For an exchangeable stochastic process $\left\{\{\mathcal{A}, \varphi\}, \mathcal{B},\left(j_{k}\right)_{k \in \mathbb{Z}}\right\}$, in the same notations introduced above, one has

$$
\mathcal{A}_{\mathcal{S}_{0}}=\mathcal{A}_{\infty} \text {. }
$$

Proof. Let us firstly prove that $\mathcal{A}_{\infty} \subseteq \mathcal{A}_{\mathcal{S}_{0}}$. By definition any element $x \in \mathcal{A}_{\infty}$ has the form

$$
x=\lim _{N \rightarrow \infty} \frac{1}{N} \sum_{l=1}^{N} u^{l}(a) ; \quad a \in \pi_{\varphi}(\mathcal{A}) .
$$

We need to prove that it is $\mathcal{S}_{0}$-invariant. In fact for any $\sigma \in \mathcal{S}_{0}$

$$
u_{\sigma}(x)=\lim _{N \rightarrow \infty} \frac{1}{N} \sum_{l=1}^{N} u_{\sigma}\left(u^{l}(a)\right) .
$$


Since $\mathcal{A}:=*-\operatorname{alg}\left(\left\{\left(j_{k}(\mathcal{B})\right)_{k \in \mathbb{Z}}\right\} \cup 1_{\mathcal{A}}\right)$, if $a \in \pi_{\varphi}(\mathcal{A})$, there exists a finite set $I \subseteq \mathbb{Z}$ and $\left(b_{i}\right)_{i \in I}$ subset of $\mathcal{B}$, such that $a=\pi_{\varphi}\left(\prod_{i \in I} j_{i}\left(b_{i}\right)\right)$. Therefore, by definition, $u^{l}(a)=\pi_{\varphi}\left(\prod_{i \in I} j_{i+l}\left(b_{i}\right)\right)$ and the set

$$
I_{\sigma}(a):=\left\{n \in \mathbb{N}: u_{\sigma}\left(u^{n}(a)\right) \notin u^{n}(a)\right\}
$$

is finite. Moreover, one has

$$
\lim _{N \rightarrow \infty}\left\|\frac{1}{N} \sum_{n \in I_{\sigma}(a) \cap\{1,2, \ldots, N\}}\left[u_{\sigma}\left(u^{n}(a)\right)-u^{n}(a)\right]\right\| \leq \lim _{N \rightarrow \infty} \frac{\left|I_{\sigma}(a)\right|}{N} M=0,
$$

where

$$
M:=\max _{n \in I_{\sigma}(a)}\left\|u_{\sigma}\left(u^{n}(a)\right)-u^{n}(a)\right\|
$$

Hence it follows that

$$
\begin{aligned}
u_{\sigma}(x) & =\lim _{N \rightarrow \infty} \frac{1}{N} \sum_{n=1}^{N} u_{\sigma}\left(u^{n}(a)\right) \\
& =\lim _{N \rightarrow \infty} \frac{1}{N}\left\{\sum_{n \in I_{\sigma}(a) \cap\{1,2, \ldots, N\}} u_{\sigma}\left(u^{n}(a)+\sum_{n \in I_{\sigma}(a)} \sum_{n\{1,2, \ldots, N\}} u_{\sigma}\left(u^{n}(a)\right)\right\}\right. \\
& =\lim _{N \rightarrow \infty} \frac{1}{N}\left\{\sum_{n \in I_{\sigma}(a) \cap\{1,2, \ldots, N\}} u_{\sigma}\left(u^{n}(a)\right)-\sum_{n \in I_{\sigma}(a) \cap\{1,2, \ldots, N\}} u^{n}(a)+\sum_{n=1}^{N} u^{n}(a)\right\} \\
& =\lim _{N \rightarrow \infty} \frac{1}{N} \sum_{n=1}^{N} u^{n}(a)=x .
\end{aligned}
$$

Thus $x \in \mathcal{A}_{\mathcal{S}_{0}}$.

Conversely, let us prove that

$$
\mathcal{A}_{\mathcal{S}_{0}} \subseteq \mathcal{A}_{\infty}
$$

Take $x \in \mathcal{A}_{\mathcal{S}_{0}}$. By definition $\mathcal{A}_{\mathcal{S}_{0}} \subseteq \pi_{\varphi}(\mathcal{A})^{\prime \prime}$ and, by the double commutant von Neumann's Theorem (see Theorem 5.3.1 of Ref. 21), $\pi_{\varphi}(\mathcal{A})^{\prime \prime}$ is the weak and strong closure of $\pi_{\varphi}(\mathcal{A})$. Then, for any $\varepsilon>0$ there exist a finite set $\left\{k_{1}, \ldots, k_{q}\right\} \subseteq \mathbb{Z}$ and $b_{1}, \ldots, b_{q} \in \mathcal{B}$ such that

$$
\left\|x-\pi_{\varphi}\left(j_{k_{q}}\left(b_{q}\right) \cdots j_{k_{1}}\left(b_{1}\right)\right)\right\|<\frac{\varepsilon}{4} .
$$

Denote $a:=j_{k_{q}}\left(b_{q}\right) \cdots j_{k_{1}}\left(b_{1}\right)$. If $u$ is the shift on $\pi_{\varphi}(\mathcal{A})$, one has

$$
\begin{aligned}
\|u(x)-x\| & \leq\left\|u(x)-u\left(\pi_{\varphi}(a)\right)\right\|+\left\|u\left(\pi_{\varphi}(a)\right)-\pi_{\varphi}(a)\right\|+\left\|\pi_{\varphi}(a)-x\right\| \\
& <\frac{\varepsilon}{2}+\left\|u\left(\pi_{\varphi}(a)\right)-\pi_{\varphi}(a)\right\|,
\end{aligned}
$$

where the last inequality follows from the fact that $u(\cdot)=U(\cdot) U^{*}$, with $U$ unitary. Notice that $u$ can be seen as a suitable $u_{\sigma},\left(\sigma \in \mathcal{S}_{0}\right)$ on $\pi_{\varphi}\left(\mathcal{A}_{\left\{k_{1}, \ldots, k_{q}\right\}}\right)$, 
where $\mathcal{A}_{\left\{k_{1}, \ldots, k_{q}\right\}}:=\prod_{k \in\left\{k_{1}, \ldots, k_{q}\right\}} j_{k}(\mathcal{B})$. Namely, denote $\bar{k}:=\max \left\{k_{1}, \ldots, k_{q}\right\}$, $\underline{k}:=\min \left\{k_{1}, \ldots, k_{q}\right\}$, take $I_{\mathbb{Z}}:=[\underline{k}, \bar{k}] \cap \mathbb{Z}$ and define $\sigma: \mathbb{Z} \rightarrow \mathbb{Z}$ as

$$
\sigma(r):= \begin{cases}r+1 & \text { if } r \in I_{\mathbb{Z}}, \\ \underline{k} & \text { if } r=\bar{k}+1, \\ r & \text { otherwise. }\end{cases}
$$

Hence $\sigma \in \mathcal{S}_{0}$ and $\left.u_{\sigma}\right|_{\pi_{\varphi}\left(\mathcal{A}_{\left\{k_{1}, \ldots, k_{q}\right\}}\right)}=u$.

Therefore

$$
\begin{aligned}
\left\|u\left(\pi_{\varphi}(a)\right)-\pi_{\varphi}(a)\right\| & =\left\|u_{\sigma}\left(\pi_{\varphi}(a)\right)-\pi_{\varphi}(a)\right\| \\
& \leq\left\|u_{\sigma}\left(\pi_{\varphi}(a)\right)-u_{\sigma}(x)\right\|+\left\|x-\pi_{\varphi}(a)\right\|<\frac{\varepsilon}{2},
\end{aligned}
$$

where we used both $u_{\sigma}(\cdot)=U_{\sigma}(\cdot) U_{\sigma}^{*}, u_{\sigma}(x)=x$. In conclusion

$$
\|u(x)-x\|<\varepsilon
$$

thus giving $x \in \mathcal{A}_{\infty}$.

It is well known (see Proposition 3.1.10 of Ref. 25) that for any fixed $\varphi$, exchangeable state on $\mathcal{A}$, if $\mathcal{A}_{\mathcal{S}_{0}}=\mathbb{C} \cdot 1$, then $\varphi$ is extremal. As a consequence, taking into account the previous result, this means that any 1-ergodic state is ergodic. Hence, in the convex of exchangeable states, all the block singleton ones are extremal.

Example 3. An exchangeable, not be block singleton, not 1-ergodic state. Let $\mathcal{H}:=\mathbf{L}^{2}\left(\mathbb{R}_{+}\right)$and take the Boolean Fock space over $\mathcal{H}$

$$
\Gamma(\mathcal{H})=\mathbb{C} \oplus \mathcal{H}
$$

with vacuum vector $\Phi:=(1, \mathbf{0})$. For any $f \in \mathcal{H}$, define the creation and annihilation operators in the usual way, i.e.

$$
\begin{aligned}
A^{+}(f): \Gamma(\mathcal{H}) & \rightarrow \Gamma(\mathcal{H}), \\
A(f): \Gamma(\mathcal{H}) & \rightarrow \Gamma(\mathcal{H}),
\end{aligned}
$$

such that

$$
A^{+}(f)\left(\begin{array}{c}
\alpha \\
g
\end{array}\right)=\left(\begin{array}{c}
0 \\
\alpha f
\end{array}\right), \quad A(f)\left(\begin{array}{l}
\alpha \\
g
\end{array}\right)=\left(\begin{array}{c}
\langle f, g\rangle_{\mathcal{H}} \\
0
\end{array}\right)
$$

with $\alpha \in \mathbb{C}, g \in \mathcal{H}$. Consider $0=k_{0}<k_{1}<\cdots<k_{n}<\cdots$ a partition on $\mathbb{R}_{+}$such that for all $i, j \geq 0, k_{i+1}-k_{i}=k_{j+1}-k_{j}=$ : $d$. For any $i \geq 0$, define the position operator $Q_{k_{i}}:=A\left(\chi_{\left[k_{i}, k_{i+1}\right)}\right)+A^{+}\left(\chi_{\left[k_{i}, k_{i+1}\right)}\right)$, where $\chi$ denotes the indicator. If

$$
\mathcal{A}:=*-\operatorname{alg}\left\{1, Q_{k_{i}}: i \geq 0\right\}, \quad \varphi:=\langle\Phi, \cdot \Phi\rangle,
$$


then $(\mathcal{A}, \varphi)$ is an algebraic probability space. Choosing $n \in \mathbb{N}, i_{1} \neq i_{2} \neq \cdots \neq i_{n}$, $i_{j} \geq 0$ for each $j=1, \ldots, n, p_{1}, \ldots, p_{n} \in \mathbb{N}$, it follows

$$
\left\langle\Phi, Q_{k_{i_{1}}}^{p_{1}} Q_{k_{i_{2}}}^{p_{2}} \cdots Q_{k_{i_{n}}}^{p_{n}} \Phi\right\rangle= \begin{cases}0 & \text { if } \exists j=1, \ldots, n \text { s.t. } p_{j} \text { is odd } \\ d^{\frac{1}{2}}\left(\sum_{j=1}^{n} p_{j}\right) & \text { otherwise }\end{cases}
$$

i.e. the mixed moments do not depend on the particular interval $\left[k_{i}, k_{i+1}\right)$ chosen. Hence, for any $\sigma \in \mathcal{S}_{0}$

$$
\varphi\left(Q_{k_{i_{1}}}^{p_{1}} Q_{k_{i_{2}}}^{p_{2}} \cdots Q_{k_{i_{n}}}^{p_{n}}\right)=\varphi\left(Q_{k_{\sigma\left(i_{1}\right)}}^{p_{1}} Q_{k_{\sigma\left(i_{2}\right)}}^{p_{2}} \cdots Q_{k_{\sigma\left(i_{n}\right)}}^{p_{n}}\right),
$$

i.e. $\varphi$ is exchangeable. Finally we prove that it is not a strict singleton state. In fact, by (3.17)

$$
\left\langle\Phi, Q_{k_{i_{1}}} Q_{k_{i_{2}}}^{2} Q_{k_{i_{1}}} \Phi\right\rangle=0 .
$$

If the state is strict singleton, one has

$$
\left\langle\Phi, Q_{k_{i_{1}}} Q_{k_{i_{2}}}^{2} Q_{k_{i_{1}}} \Phi\right\rangle=\left\langle\Phi, Q_{k_{i_{2}}}^{2} \Phi\right\rangle\left\langle\Phi, Q_{k_{i_{1}}}^{2} \Phi\right\rangle=d^{2}
$$

which contradicts (3.18). Then $\varphi$ cannot be block singleton, or by Theorem 3.2, 1-ergodic.

To obtain the equivalence between block singleton and extremal states or, equivalently, ergodicity and 1-ergodicity one needs additional requests on the pair $(\mathcal{A}, \varphi)$, such as the $\mathcal{S}_{0}$-abelianity (see Definition 3.1.11 of Ref. 25). This means that $P_{\mathcal{S}_{0}} \pi_{\varphi}(\mathcal{A}) P_{\mathcal{S}_{0}}$ is a family of mutually commutative operators, where $P_{\mathcal{S}_{0}}$ is the orthogonal projection of $\mathcal{H}_{\varphi}$ onto $E_{\mathcal{S}_{0}}$. In the classical case this condition is automatically satisfied, since $\mathcal{A}$ is a subalgebra of $\mathbf{L}^{\infty}(\Omega, \mathcal{F}, \mathbf{P})$ which is an abelian algebra. Before introducing a quantum analogue of De Finetti's theorem, we firstly present a version of the classical one, which makes use of the equivalence between strict singletons states and stochastic independence of a process.

Theorem 3.3. The classical exchangeable stochastic processes form a Choquet simplex whose extremal points are exactly those made by stochastically independent random variables.

Proof. Notice that classical exchangeable states (process) form a Choquet simplex (see Theorem 3.1.14 of Ref. 25). Moreover, in the classical case stochastic independence is equivalent to the strict singleton condition and the condition TOD is satisfied.

Thus, thanks to Proposition 3.3, any process made by stochastically independent random variables is extremal.

Conversely, for any $q \in \mathbb{N}, k_{1}, \ldots, k_{q} \in \mathbb{Z}, b_{1}, \ldots, b_{q} \in \mathcal{B}$, let us compute the mixed moment

$$
\varphi\left(j_{k_{q}}\left(b_{q}\right) \cdots j_{k_{1}}\left(b_{1}\right)\right)
$$


where $\varphi$ is extremal. As a consequence of commutativity and recalling the $j_{k}$ 's are homomorphisms, without loss of generality, we can assume that $k_{q}<k_{q-1}<\cdots<$ $k_{1}$, i.e. the $k_{i}$ 's are all singletons. Then, by Theorem 3.2 of Ref. $4,(3.19)$ is equal to

$$
\prod_{l=1}^{k} \varphi_{0}\left(b_{l}\right), \quad \varphi_{0}:=\varphi \circ j_{k_{l}}, \quad l=1, \ldots, q
$$

thus giving $\varphi$ strict singleton.

The following result and its corollaries can be seen as quantum versions of the De Finetti's theorem. Denote by $\mathcal{S}_{E}$ the set of exchangeable states.

Theorem 3.4. If the pair $(\mathcal{A}, \varphi)$ is $\mathcal{S}_{0}$-abelian, then the weak* compact convex set of exchangeable states is a Choquet simplex whose extremal points are exactly all the block singleton states. Therefore, for any $\varphi \in \mathcal{S}_{E}$, there exists a unique Radon probability measure $\mu$ on $\mathcal{S}_{E}$ such that

$$
\varphi(a)=\int_{\mathcal{S}_{E}} \psi(a) d \mu(\psi), \quad a \in \mathcal{A}
$$

and for any Baire set $\Delta$ in $\mathcal{S}_{E}$ with $\mathcal{E}_{E} \cap \Delta=\emptyset, \int_{\Delta} d \mu(\psi)=0$ (where $\mathcal{E}_{E}$ is the set of extreme points of $\mathcal{S}_{E}$ ).

Proof. The $\mathcal{S}_{0}$-abelianity of the pair $(\mathcal{A}, \varphi)$ implies, thanks to ergodic decomposition theorem (see Theorem 3.1.14 of Ref. 25), that the exchangeable states are a Choquet simplex. The same condition gives, for any $\varphi$ extremal in this simplex, $\mathcal{A}_{\mathcal{S}_{0}}=\mathbb{C} \cdot 1$ (see Proposition 3.1 .12 of Ref. 25). Hence, by Proposition 3.4, $\varphi$ is 1-ergodic. Using Theorem 3.2 one achieves that $\varphi$ is block singleton. The remaining part of the thesis is a consequence of the above mentioned ergodic decomposition theorem.

Corollary 3.4. Let us assume that $\mathcal{A}$ satisfies the $\mathcal{S}_{0}$-abelianity. If $\varphi$ is exchangeable and extremal, then $\varphi$ is a strict singleton state.

Corollary 3.5. Let us assume that $\mathcal{A}$ satisfies the condition TOD. If $\varphi$ is an exchangeable and strict singleton state, then it is extremal.

Remark 3.2. Denote by $\mathcal{S}_{B}$ the closed set of exchangeable and block singleton states, $\mathcal{S}_{S}$ the closed set of exchangeable and strict singleton states and suppose $\mathcal{A}$ satisfies the $\mathcal{S}_{0}$-abelianity. Theorem 3.4 and Krein-Milmann imply $\mathcal{S}_{E}=\overline{c o} \mathcal{S}_{B}$, where $\overline{c o}$ denotes the closed convex hull. Since $\mathcal{S}_{B}$ is contained in $\mathcal{S}_{S}$, we have $\mathcal{S}_{E}=\overline{c o} \mathcal{S}_{S}$. Hence, even if $\mathcal{S}_{B}$ and $\mathcal{S}_{B}$ are not equal, they have the same convex hull. 


\section{References}

1. L. Accardi, Y. Hashimoto and N. Obata, Notions of independence related to the free group, Infin. Dimens. Anal. Quantum Probab. Relat. Top. 1 (1998) 201-220.

2. L. Accardi, An Outline of Quantum Probability (Uspehi Math. Nauk., 1993).

3. L. Accardi, A. Frigerio and J. Lewis, Quantum stochastic processes, Publ. Res. Inst. Math. Sci. 18 (1982) 97-133.

4. L. Accardi and Y. G. Lu, A continuous version of De Finetti's theorem, Ann. Probab. 21 (1993) 1478-1493.

5. L. Accardi and G. Pistone, De Finetti's theorem, sufficiency, and Dobrushin's theory, in Exchangeability in Probability and Statistics, eds. G. Koch et al. (North Holland, 1982), pp. 125-156.

6. A. Bach, Indistinguishable Classical Particles (Springer, 1997).

7. A. Ben Ghorbal and V. Crismale, Independence arising from interacting Fock spaces and related central limit theorem, to appear in Probab. Math. Statist.

8. O. Bratteli and D. Robinson, Operator Algebras and Quantum Statistical Mechanics I, 2nd edn. (Springer, 1987).

9. H. Bühlmann, Austauschbare Stochastishe variabeln und ihre grenzwertsatze, Univ. California Publ. Statist. (1960) 31-36.

10. C. Dellacherie and P.-A. Meyer, Probabilités et Potentiel, Chaps. V and VIII (Hermann, 1980).

11. P. Diaconis and D. Freedman, A dozen de Finetti-style results in search of a theory, Ann. Inst. Henri Poincare, Sup.au N. 223 (1987) 397-423.

12. L. E. Dubins and D. A. Freedman, Exchangeable processes need not be mixtures of independent, identically distributed random variables, Z. Wahr. Verw, Gebiete 48 (1979) $115-132$.

13. M. Fannes, J. T. Lewis and A. Verbeure, Symmetric states of composite systems, Lett. Math. Phys. 15 (1988) 255-260.

14. D. A. Freedman, Invariance under mixing which generalize De Finetti's theorem, Ann. Math. Sta. 33 (1962) 916-923.

15. D. A. Freedman, Invariance under mixing which generalize De Finetti's theorem: Continuous time parameter, Ann. Math. Statist. 34 (1963) 1194-1216.

16. E. Hewitt and L. F. Savage, Symmetric measures on Cartesian products, Trans. Amer. Math. Soc. 80 (1955) 470-501.

17. A. Hora and N. Obata, Quantum Probability and Spectral Analysis of Graphs (Springer, 2007).

18. R. L. Hudson and G. R. Moody, Locally normal symmetric states and an analogue of de Finetti's theorem, Z. Wahr. Verw. Gebiete 33 (1976) 343-351.

19. R. L. Hudson, Analogues of de Finetti's theorem and interpretative problems of quantum mechanics, Found. Phys. 11 (1981) 805-808.

20. R. Jajte, Strong Limit Theorems in Non-commutative Probability, Springer Lect. Notes Math., Vol. 1110 (Springer, 1985).

21. R. Kadison and J. Ringrose, Fundamentals of the Theory of Operator Algebras I (Academic Press, 1983).

22. O. Kallenberg, Characterizations and embedding propertiers in exchangeability, Tech. Rep. 10 (1981).

23. C. Köstler, A noncommutative extended De Finetti's theorem. (electronic) arXiv: 0806.3621v1 [math.OA].

24. F. Lehner, Cumulants in Noncommutative Probability III. Creation and annihilation operators on Fock spaces, Infin. Dimens. Anal. Quantum Probab. Relat. Top. 10 (2007) 335-363. 
660 L. Accardi et al.

25. S. Sakai, $C^{*}$-algebras and $W^{*}$-algebras (Springer, 1971).

26. M. Schürmann, White Noise on Bialgebras, Lect. Notes Math., Vol. 1544 (SpringerVerlag, 1993).

27. E. Störmer, Symmetric states of infinite tensor products of $\mathbf{C}^{*}$-algebras, J. Funct. Anal. 3 (1969) 48-68.

28. H. Umegaki, Conditional expectation in an operator algebra, Tohoku Math. J. 6 (1954) 177-181. 